\title{
Load Calculation and Design of Roller Crowning of Truck Hub Bearing
}

\author{
Xintao Xia ${ }^{*}, 1$, Shujing Dong ${ }^{1}$, Liming Sun $^{2}$ and Tianju Chen ${ }^{3}$ \\ ${ }^{1}$ Henan University of Science and Technology, Luoyang, 471003, P.R. China \\ ${ }^{2}$ Luoyang Bearing Research Institute, Luoyang, 471003, P.R. China \\ ${ }^{3}$ Pittsburg State University, Pittsburg, KS 66762, USA
}

\begin{abstract}
Double-row tapered roller bearing 353112 is used in trucks, and the external load and pressure distribution of the bearing are calculated combining with the specific operating conditions. Tapered roller bearings typically employ roller profile modification to equalize the load distribution, minimizing the stress concentration at the roller ends. Doing FEA (finite element analysis) on the contact stress with the changing roller crowning between roller and races, the appropriate roller profile and the crown drop of roller can be achieved. Finally the best control equation of roller crowning is obtained. It has important guiding significance for the improvement of roller crowning design of truck hub bearings.
\end{abstract}

Keywords: Hub bearings, load distribution, roller crowning, stress analysis.

\section{INTRODUCTION}

The hub bearing is an important component for transmitting movement and force in the truck. Typically truck wheel bearings require tapered roller bearings to operate under heavy combined radial and thrust loading at varying operating conditions. The topic discussed in this paper is the force situation and roller crowning of doublerow tapered roller bearing 353112 in the axles of trucks.

Life of bearings has been a subject of considerable interest to both bearing manufactures and designers. The improvement of bearing life has always been a concerned issue, which not only involves bearing materials and manufacturing level, but also involves the loading situation [1-4] and design scheme of bearings, especially generatrix profile selection [5] and the crown drop of rollers. The stress concentration should be avoided in elastic contact problems because failure would occur at the ends [6,7]. The contribution of roller crowning to the bearing life has been studied considerably by the researchers $[8,9]$. In this paper external load and load distribution of the truck hub bearing are calculated in terms of the specific working conditions. According to the technical parameters of the bearing, the crown drop of roller can be obtained by combining the operating conditions, FEM analysis and optimal roller profile.

\section{CALCULATION OF THE TRUCK HUB BEARING}

\subsection{Calculation of External Load of the Bearing}

External load of hub bearing is transmitted from the wheels to the hub bearing.

Taking the end of the larger force of truck hub bearing as research object, the radial force and axial force are given as [10]:

*Address correspondence to this author at the Henan University of Science and Technology, 48 Xiyuan Road, Luoyang, Postcard: 471003, P.R. China; Tel: +86-1394929 7723; E-mail: xiaxt1957@163.com

$$
\begin{aligned}
& F_{\mathrm{r}}=f_{\mathrm{w}}\left(\frac{1}{2} W g+\frac{h}{L_{h}} W a\right) \\
& F_{\mathrm{a}}=-F_{\mathrm{r}} \frac{a}{g}
\end{aligned}
$$

where $F_{\mathrm{r}}$ is the radial force, $F_{\mathrm{a}}$ is the axial force, $W$ is the mass of rear axle on the truck, $g$ is gravitational acceleration, $h$ is the height of the center of the mass, $L_{h}$ is the distance of rear wheels, $a$ is the lateral acceleration, which can be described by the multiple of $g$, and generally taking a maximum of $0.55 \mathrm{~g}, f_{\mathrm{w}}$ is the impact coefficient for the force due to different working conditions, and $(-)$ indicates the axial force which keeps opposite direction with that of the lateral acceleration. Structure parameters of the truck are shown in Table 1.

Table 1. Structure parameters of the truck.

\begin{tabular}{|c|c|c|c|c|}
\hline Structure Parameters & $\boldsymbol{W}$ & $\boldsymbol{L}_{\boldsymbol{h}}$ & $\boldsymbol{f}_{\mathrm{w}}$ & $\boldsymbol{h}$ \\
\hline \hline value & $4.5 \mathrm{t}$ & $1590 \mathrm{~mm}$ & 1 & $898 \mathrm{~mm}$ \\
\hline
\end{tabular}

Through equations (1) and (2), we get:

$F_{\mathrm{r}}=35749 \mathrm{~N}, F_{\mathrm{a}}=-19662 \mathrm{~N}$

\subsection{Calculation of Theory Load Distribution of the Roller}

Bearing for the truck is the double-row tapered roller bearing 353112, and its technical parameters are shown in Table 2. Two rows of rolling elements are under nonuniform load after the bearing is subjected to radial and axial loads. For roller bearings, if $F_{\mathrm{a}} \geq 1.909 F_{\mathrm{r}} \tan \alpha$, then the loading process is only on one roller column of the bearing. Load distribution can be calculated as a single-row tapered roller bearing $[11,12]$. 
Table 2. Technical Parameter of the bearing.

\begin{tabular}{|c|c|}
\hline Technical Parameter & Value \\
\hline Outer cone angle, $\alpha$ & $14.8^{\circ}$ \\
\hline Inner cone angle, $\beta$ & $11.7^{\circ}$ \\
\hline Flange angle, $\gamma$ & $77.95^{\circ}$ \\
\hline Small end diameter of roller, $d_{1}$ & 9.061 \\
\hline Large end diameter of roller, $d_{2}$ & 10.258 \\
\hline Number of rollers in the bearing, $z$ & $22 \times 2$ \\
\hline Effective length of roller, $l_{\mathrm{w}}$ & 20.65 \\
\hline Length of roller, $l$ & 21.65 \\
\hline Inner diameter, $d$ & 60 \\
\hline Outer diameter, $D$ & 102 \\
\hline Bearing width, $B$ & 90 \\
\hline
\end{tabular}

The bearing meets the condition of $F_{\mathrm{a}} \geq 1.909 F_{\mathrm{r}} \tan \alpha$, so load distribution could be calculated as a single-row tapered roller bearing. It is necessary to establish the loaddisplacement relationship of the rolling elements with their races to solve the load distribution. The following assumptions are also made:

(1) Generally the speed of the truck bearing is not particularly high, so the centrifugal force and gyroscopic moment produced by the bearing are very small. That will not have a significant impact on load distribution of the roller. Centrifugal force and gyroscopic moment are considered absent at all contacts.

(2) There is a permanent contact causing stress along the entire length of the tapered roller; friction force of the roller is ignored.

(3) Friction moments of the roller are neglected.

For roller bearings, the relationship of load- displacement can be written as:

$Q=K \delta^{n}$

where $n=1.11$ and $K=7.86 \times 10^{4} l^{8 / 9}$

Having been subjected to radial and axial forces, the relative displacement of inner and outer of tapered roller bearings in the radial and axial are respectively $\delta_{\mathrm{r}}$ and $\delta_{\mathrm{a}}$. Stipulating the position of the maximum load for the roller is $\theta=0$, where $\theta$ is position angle of rollers. The total deflection in the arbitrary angle location is given by:

$\delta_{\theta}=\delta_{\mathrm{a}} \sin \alpha+\delta_{\mathrm{r}} \cos \theta \cos \alpha$

When $\theta=0$, the maximum deflection is given as:

$\delta_{\max }=\delta_{\mathrm{a}} \sin \alpha+\delta_{\mathrm{r}} \cos \alpha$

From equations (4) and (5), $\delta_{\theta}$ can be obtained as follows:

$\delta_{\theta}=\delta_{\max }\left[1-\frac{1}{2 \varepsilon}(1-\cos \theta)\right]$ where,

$\varepsilon=\frac{1}{2}\left(1+\frac{\delta_{\mathrm{a}}}{\delta_{\mathrm{r}}} \tan \alpha\right)$

The angle of load distribution is therefore:

$\theta_{1}=\cos ^{-1}(1-2 \varepsilon)$

By equation (3), roller load of the arbitrary position can be written as:

$Q_{\theta}=Q_{\max }\left[1-\frac{1}{2 \varepsilon}(1-\cos \theta)\right]^{n}$

By Introducing radial integral $J_{\mathrm{r}}(\varepsilon)$ and axial integral $J_{\mathrm{a}}(\varepsilon)$ of the load distribution, the equilibrium equation of the bearing is expressed as:

$F_{\mathrm{r}}=Q_{\max } Z J_{\mathrm{r}}(\varepsilon) \cos \alpha$

$F_{\mathrm{a}}=Q_{\max } Z J_{\mathrm{a}}(\varepsilon) \sin \alpha$

where

$J_{\mathrm{r}}(\varepsilon)=\frac{1}{2 \pi} \int_{-\theta_{1}}^{+\theta_{1}}\left[1-\frac{1}{2 \varepsilon}(1-\cos \theta)\right]^{n} \cos \theta \mathrm{d} \theta$

$J_{\mathrm{a}}(\varepsilon)=\frac{1}{2 \pi} \int_{-\theta_{1}}^{+\theta_{1}}\left[1-\frac{1}{2 \varepsilon}(1-\cos \theta)\right]^{n} \mathrm{~d} \theta$

From equations (10) and (11), the maximum load for roller is given as:

$Q_{\max }=\frac{F_{\mathrm{r}}}{z J_{\mathrm{r}}(\varepsilon) \cos \alpha}=\frac{F_{\mathrm{a}}}{z J_{\mathrm{a}}(\varepsilon) \sin \alpha}$

Thus the maximum load for roller can be calculated. The loading area and roller load are shown in Fig. (1).

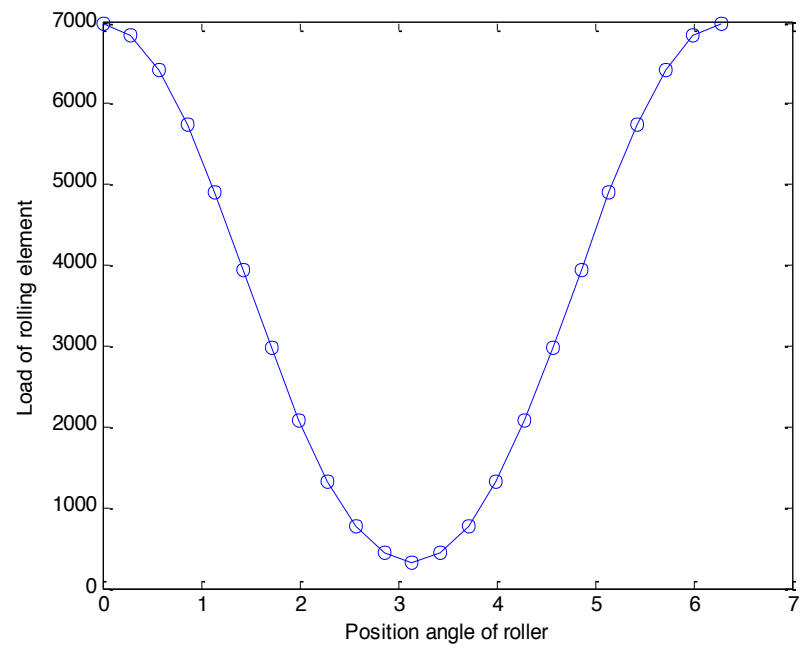

Fig. (1). Load of roller of the arbitrary position.

\section{FINITE ELEMENT MODEL OF THE TRUCK HUB BEARING 353112}

In the hub bearing, the feature of contacts of rollers and their races is completely consistent. Therefore using a local 
contact model of a roller and race, the overall roller crowning simulation can be analyzed [13, 14]. For roller modeling, the generatrix was generated in the CAD software in the form of parametric equation and then imported in ANSYS to build the model. Then, by setting the material properties, performing meshing, setting up the contact pairs and constraint conditions and applying the load, the maximum load of the roller has been calculated in the previous section that is applied on the model. After all the pre-analysis conditions are met, the operation can be performed. Minimum grid unit is $0.025 \mathrm{~mm}$. Finite element model is shown in Fig. (2).

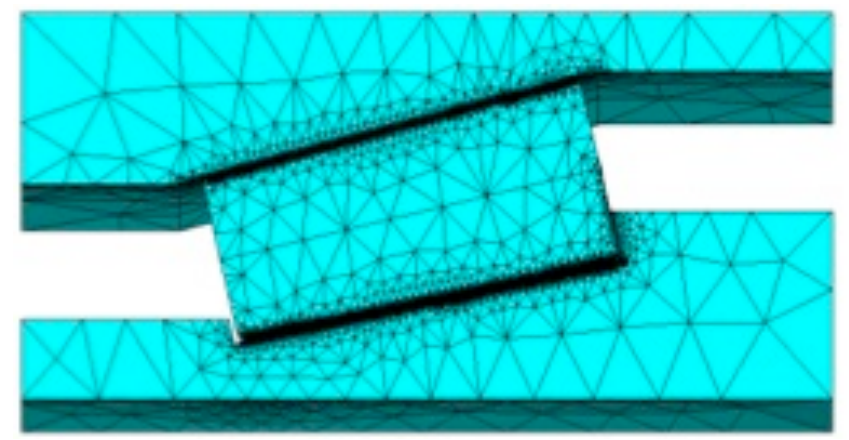

Fig. (2). Finite element model.

\subsection{The Analysis of Results}

The identical modeling method is employed. The mechanical behavior of straight generatrix shape roller and logarithmic generatrix roller are analyzed, respectively. By the theoretic calculation, a set of the crown drop of roller is accepted as: $0.005 \mathrm{~mm}, 0.01 \mathrm{~mm}, 0.015 \mathrm{~mm}$, and $0.02 \mathrm{~mm}$, and the bearing material is $\mathrm{GCr} 15$. The extreme value of contact stress between roller and their races is shown in Figs. $(3,4)$.

Fig. (5) gives the extreme values variation of contact stress of different roller crowning and large rib of the inner, and the maximum value is only $400.7 \mathrm{MPa}$.

Fig. (3) describes the variation of extreme values of contact stress between different roller crowning and inner race of the bearing. Fig. (4) is the variation of extreme values of contact stress between different roller crowning and outer race. From Figs. $(\mathbf{3}, \mathbf{4})$, it is observed that when the crown drop of roller is zero, the maximum value of contact stress can be obtained and the stress of roller and inner race is greater than that of between the roller and outer race. Before the crown drop is less than $0.005 \mathrm{~mm}$, the extreme stress decreases quickly with the increase of crown drop. This is because the phenomenon of stress concentration weakens gradually as the crown drop increases, and contact stress is distributed uniformly. When the crown drop of roller is more than $0.015 \mathrm{~mm}$, the extreme value of stress begins to rise, but the rate of increase is much smaller than the rate of descent whilst the roller-race contact surface decreases with the increase of crown drop of roller and the intermediate contact stress begins to increase.

Contact stress of different roller crowning and large rib of the inner in the Fig. (5) are quite smaller than that in the Figs. $(3,4)$. Thus we take no account of it in this paper.

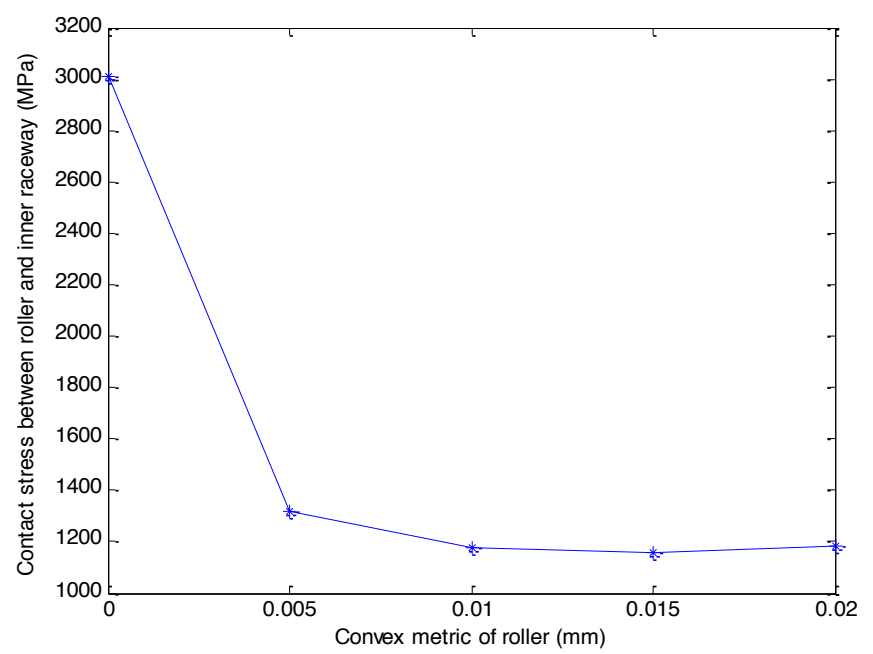

Fig. (3). The extreme values variation of contact stress between different roller crowning and inner race.

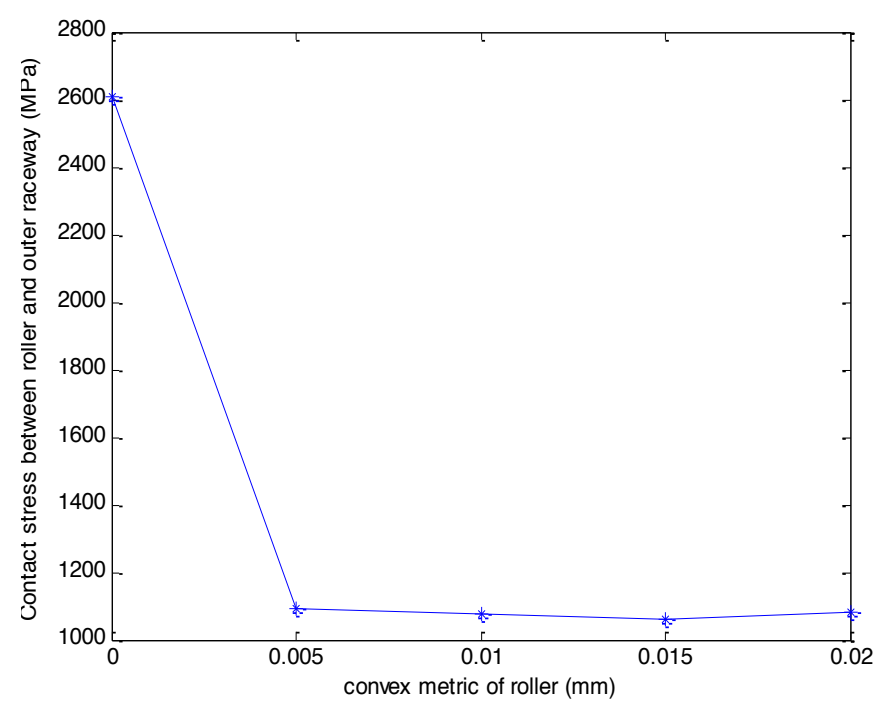

Fig. (4). The extreme values variation of contact stress between different roller crowning and outer race.

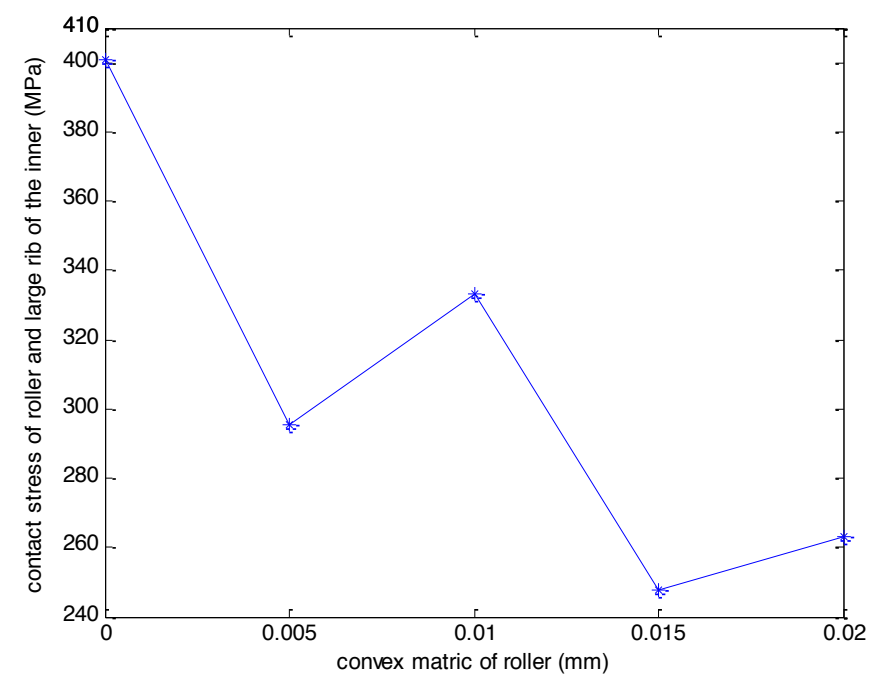

Fig. (5). The extreme values variation of contact stress between different roller crowning and large rib of the inner. 


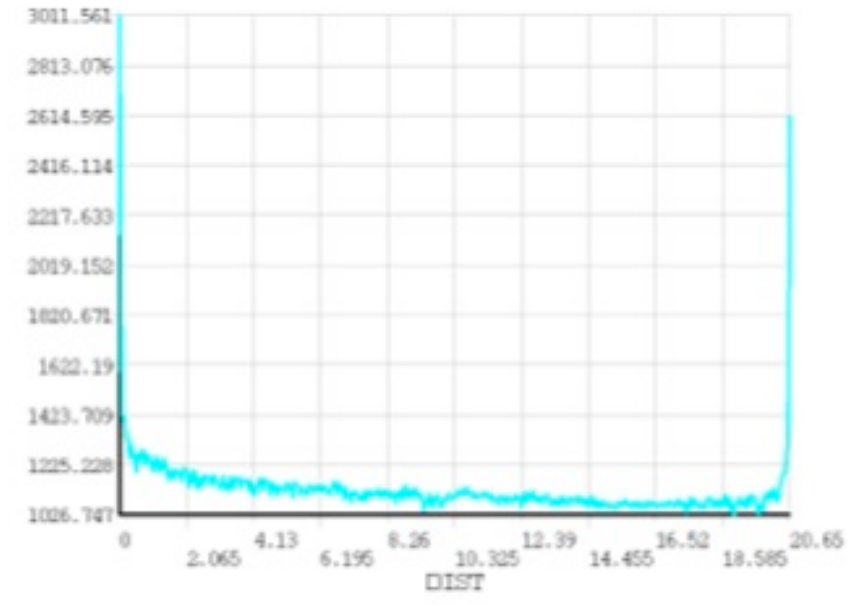

Fig. (6). Contact stress of roller of the straight generatrix and inner race.

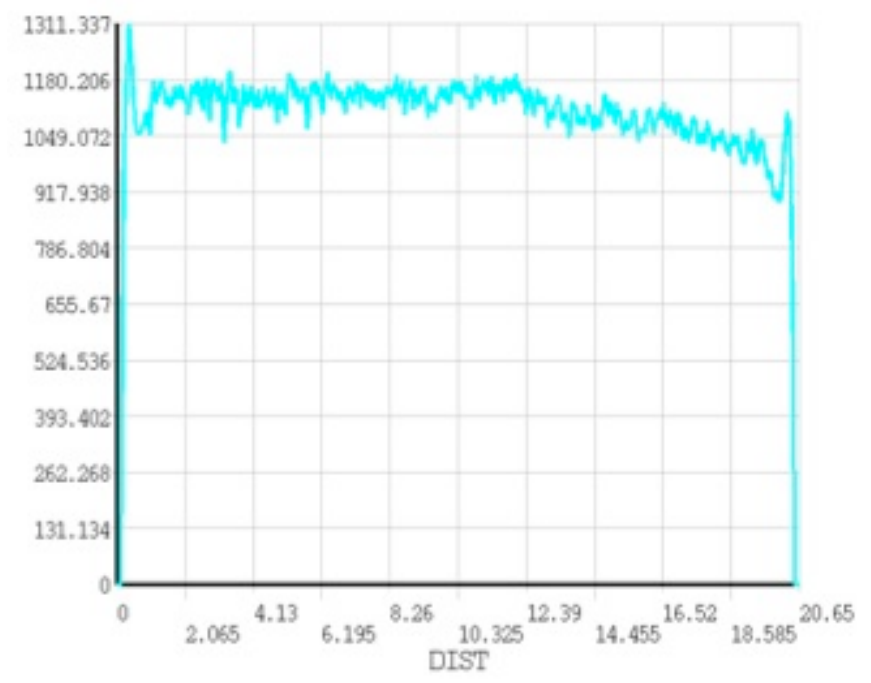

Fig. (7). Contact stress of roller crowning of $0.005 \mathrm{~mm}$ and inner race.

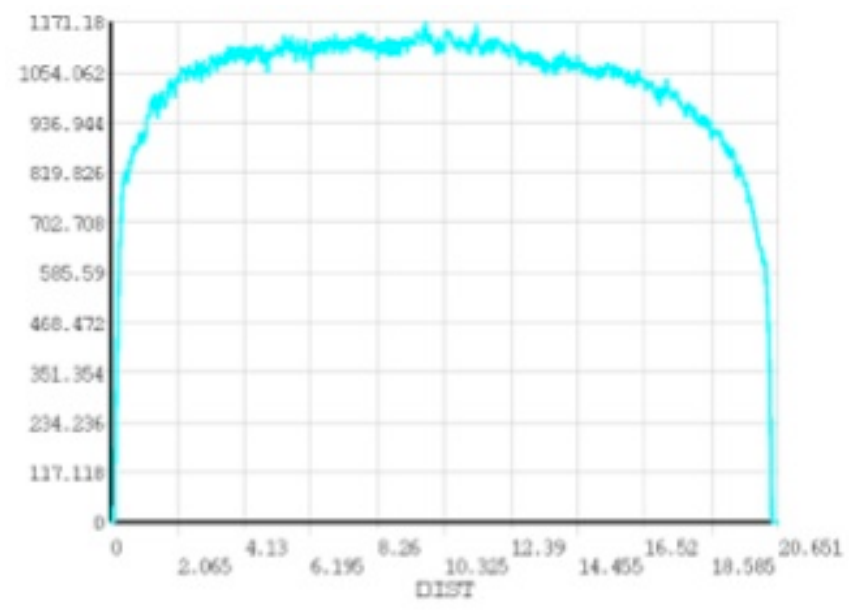

Fig. (8). Contact stress of roller crowning of $0.01 \mathrm{~mm}$ and inner race.

The contact stress of different roller crowning and inner race along the roller generatrix direction is shown from Figs. (6-10). In the figures, abscissa is the effective length of roller, and the unit is $\mathrm{mm}$. Ordinate is contact stress of roller and races, and the unit is MPa. Fig. (6) shows that straight generatrix roller has the larger contact stress at the ends of roller. Fig. (7) shows that when roller crowning is $0.005 \mathrm{~mm}$, the ends of roller have obvious stress mutation. Figs. $(\mathbf{8}, \mathbf{9})$ show, respectively, that when roller crowning is of $0.01 \mathrm{~mm}$ and $0.015 \mathrm{~mm}$, the contact stress is maintained uniformly along the direction of roller generatrix but begins to decrease gradually at the ends of roller. Fig. (10) shows that when roller crowning is $0.02 \mathrm{~mm}$, the variation curve of contact stress steepens and the roller-race contact surfaces decrease by degrees. So under this working condition, the optimal crown drop of roller is $0.01 \mathrm{~mm}$ to $0.015 \mathrm{~mm}$ and the best is close to $0.015 \mathrm{~mm}$.

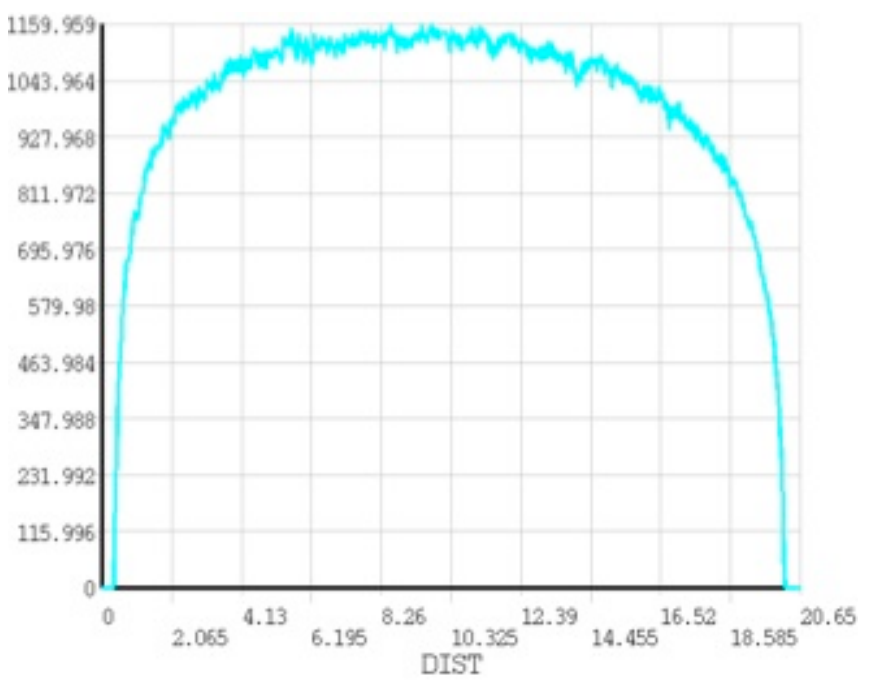

Fig. (9). Contact stress of roller crowning of $0.015 \mathrm{~mm}$ and inner race.

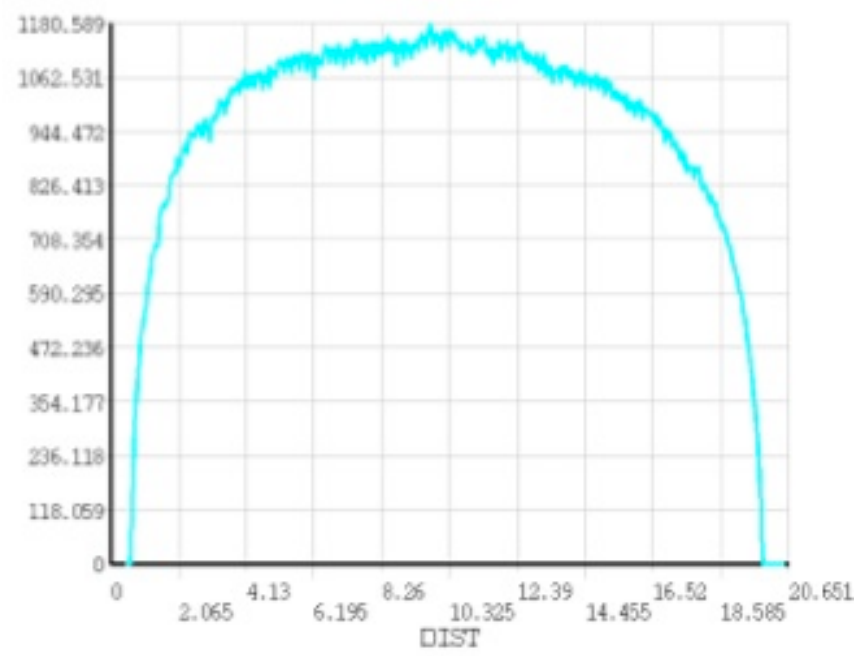

Fig. (10). Contact stress of roller crowning of $0.02 \mathrm{~mm}$ and inner race.

Through data fitting, the equation of roller crowning can be given as:

$y=-9.906 \times 10^{-4} \ln \left(1-0.00938 x^{2}\right) \quad($ when convex metric is $0.01 \mathrm{~mm}$ )

or

$y=-14.8594 \times 10^{-4} \ln \left(1-0.00938 x^{2}\right) \quad$ (when convex metric is $0.015 \mathrm{~mm}$ ) 
where, $y$ is the crown value $(\mathrm{mm})$, the direction of $x$-axis is in agreement with tangent direction of the point of maximum crowning value of roller generatrix. The range of $x$ is $|x|<10.325 \mathrm{~mm}$. Finite element analysis methods have not considered the influence of crown value of inner and outer.

\section{CONCLUSION}

According to the specific working condition, the force situation of the truck hub bearing $35311-2 \mathrm{R}$ is achieved, and the theoretical analysis of force distribution of bearing is studied. Simultaneously, the finite element analysis software is applied to model and stress of the bearing is analyzed. Furthermore the optimal value of the crown drop of roller and corresponding equations are concluded. The above equation of roller crowning is derived only for this truck hub bearing 353112, which does not have universal applicability for other types of bearings.

\section{CONFLICT OF INTEREST}

The authors confirm that this article content has no conflict of interest.

\section{ACKNOWLEDGEMENTS}

This project is supported by National Natural Science Foundation of China (Grant Nos. 51475144 and 51075123).

\section{REFERENCES}

W. Wang, F. Lin, and Y. W. Zhang, "Car wheel hub bearing mechanics performance analysis", Machinery Design and Manufacture, vol. 3, pp. 192-195, March 2014 (in Chinese).
[2] Y. Xu, Y. P. Li, and D. Hong, "Analysis of the calculation and the modes of breakdown of automobile hub bearing", Modern Manufacturing Technology and Equipment, vol. 6, pp. 49-50, 2009 (in Chinese).

[3] M. Y. Li, and K. Q. Wu, "Load test and load spectrum of auto hub bearing", Bearing, vol.6, pp.25-28, 1998 (in Chinese).

[4] L. Wang, Q. C. Wang, and Q. X. Pang, "The stress analysis of hub bearing based on multi- bench test load", Agricultural equipment and vehicle engineering, vol. 6, pp. 11-14, 2009 (in Chinese).

[5] L. Z. Yongjing, "The numerical solution of contact stress analysis of crown roller bearing", Foreign Bearing, vol. 5, pp. 16-18, 1988.

[6] Z. W. Wang, L. Q. Meng, and W. S. Hao, "Finite element method analysis and optimal design of roller convexity of tapered roller bearing", Advanced Materials Research, vol. 139-141, pp. 10791083, 2010.

[7] H. Rahnejat, and R. Gohar, "Design of profiled taper roller bearings", Tribology, vol. 12, no. 6, pp. 269-275, 1979.

[8] K. Nikpur, and R. Gohar, "Profiled taper rollers", Tribology, vol.12, no.6, pp. 203-208, 1975

[9] F. B. Oswald, E. V. Zaretsky, and J. V. Poplawski, "Effect of roller geometry on roller bearing load-life relation", Tribology Transactions, vol.57, pp.928-938, 2014.

[10] D. J. Huang, Z. Y. Weng, H. W. You, D. Yin, G. Gao, and J. Mei, "Method research of establishing load spectrum on durability test of the car hub bearing", International Conference on Mechanic Automation and Control Engineering, 2010, pp. 3132-3136.

[11] S. E. Deng, Q. Y. Jia, and Y. S. Wang, Rolling Bearing Design Principle, Bejing: Chinese Standards Press, 2008 (in Chinese).

[12] L. Hu, W. Z. Wang, and Z. Q. Zhao, "Lubricated contact analysis of roller large end-flange in double-row tapered roller bearing", $J$. Tribology, vol. 1, pp.22-28, 2013 (in Chinese).

[13] X. Y. Chen, and J. J. Ma, "Some problems in the design of convex metric of logarithmic roller", Bearing, vol. 11, pp. 2-4, 1994 (in Chinese).

[14] W. W. Li, X. Y. Chen, and X. J. Shen, "Numerical calculation and roller pressure analysis of theoretical logarithm profile equation", China Mechanical Engineering, vol.15, pp.1788-1792, 2011 (in Chinese). 